\title{
Estudios culturales y educación: Posibilidades, urgencias y limitaciones ${ }^{1}$
}

Eduardo Restrepo ${ }^{2}$

"Todos escribimosy hablamos desde un lugar y un momento determinados, desde una historia y una cultura específicas. Lo que decimos siempre está "en contexto", posicionado [... ] todo discurso está "situado", y que el corazón tiene sus razones"

Stuart Hall ([1990] 2010: 349).

En diferentes sitios de A mérica L atina, los estudios culturales tienden a generar múltiples desconciertos. En algunos lugares la relación predominante con los estudios cultural es es una de mucho escozor y tension porque se les asocia y se les atribuye las más diversas ligerezas, cuando no la simple expresion de una moda intelectual importada, En otros, se les abraza cual si fuesen panacea. Hay muchos miedos e imaginarios respecto a los estudios culturales, pero tambien un monton de seducciones y embrujos.

Los estudios culturales no son nombrados ni se los hace con las mismas implicaciones en los diferentes países. En Argentina, por ejemplo, es una etiqueta que no esta institucionalizada y con la cual parece que pocos se sienten comodos. En Colombia el asunto es distinto; sólo en Bogotá, en los últimos años se han creado maestrias de estudios culturales en tres de las más visibles universidades del pais: la Nacional, la Javeriana y la de, Los Andes, respectivamente. Otros programas de maestria o doctorado, en éstas u otras universidades, apelan al nombre de estudios culturales para nominar

1 Ponencia presentada en el 40 Seminário Brasileiro de Estudos Culturais e Educação y al 10 Seminário Internacional de Estudos Culturais e Educação (ULBRA-Canoas/RS). Puerto Alegre, Brasil. 25 de mayo de 2011. Agradezco los clarificadores comentarios de Constanza M endoza de la Universidad Pedagógica de Colombia sobre la relación entre estudios culturales y educación. He utilizado muchas de sus planteamientos, aunque los problemas de interpretación son de toda mi responsabilidad.

2 Profesor Asociado, del Departamento de Estudios Culturales Universidad Javeriana, Bogotá Colombia. 


\section{EdracbPestrepo}

Estudios culturales y educación:

Posibilidades, urgencias y limitaciones, artículo de reflexión

cursos, líneas de investigación o posibles temáticas de trabajo. En congresos, publicaciones y presentaciones de las distintas ciencias sociales y humanidades aparece, cada vez con mayor frecuencia, el amenazante o seductor espectro de los estudios culturales.

Así, en Colombia la institucionalización de los estudios culturales no es una posibilidad a ser discutida, sino que es un hecho estableccido. Independente de que nos guste o no, los programas de maestria ya estan funcionando y la etiqueta interpela cada vez, mas las subjetividades y discursos que,circulan en el establecimiento académico. Dada la institucionalizacion mencionada y las subjetividades en juego, en el caso de Colombia no estariamos tanto en el momento de definir si la etiqueta sería relevante o no, como en el de disputar su contenido. Que tipo de estudios culturales son los que vamos a construir y cuál es los proyectos intelectuales y pol íticos que vamos a establecer asociados con los estudios culturales, son las interrogantes que se abren en nuestro caso.

Para los propósitos de esta ponencia no sólo voy a plantear los criterios y caracteristicas que definirian unos estudios culturales relevantes en el contexto colombiano, sino que intentare hacerlo pensando en las posibilidades, urgencias y limitaciones de esos estudios culturales con los estudios criticos de la educación.

\section{Posibilidades}

Existe una tendencia a equiparar los estudios culturales con estudios sobre la cultura. Esta equiparación es una confusion bastante extendida, incluso entre al gunos de quienes se dicen sus practicantes. Para comprender la especificidad del proyecto intelectual y politico de los estudios cultural es es fundamental establecer una distincion entre los estudios culturales y los estudios sobre la cultura. No es suficiente con estudiar la cultura o lo cultura para hacer estudios culturales. A unque de cierta manera los estudios culturales 'estudian' la cultura o lo cultural esto no significa que cualquier estudio sobre la cultura o lo cultural puede ser adecuadamente considerado como estudios culturales.

A sumir que cualquier estudio de la cultura es automáticamente estudios culturales sería subordinar a los estudios culturales una serie de tradiciones disciplinarias o campos interdisciplinnarios que tienden a tener su especificidad y genealogías diferenciables. Por ejemplo, no podemos desconocer el enorme y muchas veces valioso trabajo adelantado desde la antropologia cultural, la sociología de la cultura la critica cultural o la historia cultural afirmando que todos esos abordajes sobre la cultura o lo cultural tendrian que ser englobados felizmente por los estudios culturales. Los estudios culturales son mucho menos, pero también al go distinto.

Los estudios culturales se diferenciarían de los estudios sobre la cultura por la combinacion de una serie de rasgos o caracteristicas que los configurarian como un 
Revista de investigaciones UNAD

Volumen 10. Número 1. Junio 2011

proyecto intelectual y politico muy particular. El primer rasgo se refiere a la forma cómo los estudios culturales entienden la categoría misma de cultura. Para los estudios cultural es la cultura se entiende en su relación mutuamente constitutiva con el poder, de ahí que hablen de la cultura-como-poder, pero también del podercomo-cultural.

Para elaborar este punto es útil partir de la distinción de tres tipos de definiciones de la cultura que hace N elly Richard (2001). L a definición antropológica, subraya Richard, es aquella que conșidera a la cultura como modo o forma de vida. Siguiendo a B ourdieu y la sociologia de la cultura, Richard considera que hay una segunda definición que supone lo cultural como un campo de relaciones sociales institucionalizadas con su, especificidad y cierta autonomia. Finalmente, la definicion que Richard asocia con los estudios culturales (en la tradicion GramsciWilliams-Hall) es aquella que introduce en la concepción misma de lo cul tural la problemática del poder y la hegemonia.

Independientemente de que estemos de acuerdo o no con estas distinciones, lo que me interesa subrayar aqui para la cartografia de los estudios culturales es que la palabra de cultura ha encerrado muchos conceptos distintos (y hasta antagonicos) no solo entre los diversos campos disciplinarios y transdisciplinarios, sino al interior de los mismos. Para referir brevemente a la antropologia, no es lo mismo la categoria de cul tura que propuso Tyl or a finales del siglo X IX como la totali dad depracticas, costumbres, creencias, tecnologias, etc... de la que planteaba Clifford Geertz en los años sesenta de la cultura como una telaraña de significados en las cuales los seres humanos estamos suspendidos.

Cuando se habla de cultura en estudios cultural es necesariamente nos referimos a la cultura-como-poder y al poder-como-cultura, Y aunque no se puede sugerir que únicamente los estudios culturales operan desde una categoria de cultura de esta naturaleza, si se puede afirmar que no es concebible la existencia de unos estudios culturales que no operen en el amplio marco abierto por esta categoria.

Estrechamente ligado a esta conceptualización de la cultura se puede indicar otro rasgo o criterio de distincion de los estudios culturales. Es comun escuchar el planteamiento de que los estudios culturales no son una disciplina, sino que son un campo interdisciplinario o, mejor aun, transdisciplinario (para algunos, incluso, son no disciplinarios o indisciplinados). ${ }^{3}$

Cuando Stuart Hall (2007), por ejemplo, se refiere a los estudios culturales como un campo interdisciplinario estaba pensando no en la desaparición de las disciplinas, ni que los estudios culturales eran una suerte de superacion de estas (como arrogantemente al gunos practicantes de estudios culturales lo predican), sino que para entender los complejos amarres de lo cultural y el poder en lo

3 Ver, por ejemplo, el volumen editado por Catherine Walsh, Freya Schiwy y Castro-Gómez (2002). 


\section{EdracbPestrepo}

Estudios culturales y educación

Posibilidades, urgencias y limitaciones, artículo de reflexión

concreto se requería de una labor intelectual no reduccionista. Los estudios culturales se constituyen como un pensamiento no reduccionista.

El reduccionismo consiste en reducir a una variable o aspecto analítico (lo economico, lo social, lo cultural o lo discursivo) las 'explicaciones' ofrecidas.

El economicismo es un buen ejemplo del reduccionismo: de antemano el investigador sabe que la causa que explica todo es la economía. Lo mismo pasa con el sociologicismo, el historicismo, el cultural ismo o el discursivismo: se sabe que son las relaciones sociales, la Historia (asi con mayúscula), lo cultural 0 el discurso respectivamente lo que determina y explica.

Cyando se opera desde un reduccionismo como el culturalismo. (en el cual han caido muchos antropologos), no solo todos los otros aspectos o niveles analiticos de la vida social que no son considerados cultura se explican en terminos culturales, sino que la misma cultura es explicada cultural mente. Por eso se puede afirmar que el reduccionismo es un pensamiento con garantías'. siempre sabe cuál es la respuesta (aś sea bajo piruetas como las de 'en ultima instancia'), y las investigaciones empiricamente orientadas no tienen otra funcion que la demostrar lo que ya se sabla de antemano.

En un abierto contraste con el reduccionismo, Hall ([1992] 2010) considera que los estudios culturales son no reduccionistas. No asumen de antemano explicaciones unidimensionales ni las reducen a una variable 0 aspecto universalizado como garante ultimo de un sistema teorico totali itarista. Los estudios culturales han reaccionado al economicismo e historicismo presente en las versiones del marxismo vul gar, pero tambien han reaccionado al cultural ismo de las versiones de la antropol ogla mas, convencional 0 al textualismo de las versiones postmodernas y banales de la teoria social contemporanea.

Dela misma manera, lainvestigación no es entendidacomo lasimple corroboración de angelicales formulaciones teoricas definidas de antemano, sino como la ardua y honesta labor de comprender la especificidad y densidad de lo concreto en un ejercicio que implica una necesaria conceptualización que no evita poner en cuestion los postulados teóricos desde los que se opera. Por tanto, siguiendo la feliz formulacion de Stuart Hall (2010), los estudios culturales se consitituyen en un pensamiento sin garantías.

En los estudios culturales lainterdisciplinariedad nace como un esfuerzo intelectual para ir mas alla del reduccionismo que tiende a ser sentido comun disciplinario. Hay que hacer 'mejor' sociologia de la que suelen hacer los sociologos,o 'mejor' antropologia de la que tienden a producir los antropologos... y asI sucesivamente. 'M ejor' en el sentido de esforzarse por comprender lo que estos practicantes de las disciplinas hacen desde preguntas y asumiendo riesgos que son pertinentes a los estudios cultural es. No es la arrogancia comoda de predicar (usualmente desde una escandal osa ignorancia) que estas disciplinas estan 'superadas' o 'mandadas a 
Revista de

investigaciones UNAD

Volumen 10. Número 1. Junio 2011

recoger'. Es la invitación a rearticular creativa y críticamente, aquellos aspectos conceptuales o metodologicos de origenes disciplinarios multiples (o incluso de campos transdisciplinarios o no disciplinarios como la teoria feminista) en función de las preguntas y en el estilo propio de los estudios culturales.

La razón por la cual los estudios culturales constituyen un pensamiento sin garantias no es simplemente porque de esta forma se puede producir conocimiento más adecuado sobre el mundo de la vida social (en el sentido de interpretaciones mas ajustadas a la compleja densidad de lo concreto), sino fundamental mente porque los estudios cul tural es han sido desde siempre pensados como un proyecto con una abierta voluntad política Al respecto, suele citarse la once tesis sobre Feuerbach de M arx de que refiere como los filosofos han interpretado de diferentes maneras el mundo, lo que importa sin embargo es transformarlo. Los estudios culturales pretenden transformar el mundo (claro que de formas muy distintas a como la izquierda ortodoxa lo ha concebido) y consideran que el conocimiento y la teoria son herramientas, e importantes terrenos de disputa para lograrlo. Una inadecuada interpretacion del mundo, suele conducir a una erratica politica; aunque, hay que decirlo, una mas adecuada interpretacion no es tampoco garantía del exito en la intervencion política.

Obviamente, los estudios culturales, no son el único campo que encuentra su proposito en la intervencion política y que, por tanto, cuestiona el caro principio de las diversas vertientes del pensamiento positivista y cientificista de que los hechos y los valores, el conocimiento y la política (o la ética) el objeto y el sujeto, el mundo y la palabra, son ontologicamente independientes. Pero no son imaginables unos estudios culturales que abracen modalidades de positivismo o que se conciban como unos simples productores de conocimiento desconectado de su para qué, de las dimensiones politicas y éticas (y esto no como posterioridad o exterioridad, sino como inmanentes y su razon de ser).

Es,por esto que los estudios cultural es nunca son sólo estudios, siempre son algo mas. Es por esto que no se circunscriben a la academia. No es un conocimientoflorero un conocimiento ostentoso, lo que buscan producir los estudios culturales, Lo que no significa, sin embargo, que los estudios culturales sean anti-academicos 0 anti-teóricos. No son un llamado a abandonarle a la derecha (o a los positivistas) la academia y la teoría desde la fal sa premisa populista que el 'verdadero conocimiento' esta en otro lado o del ingenuo supuesto de que el ciego activismo ofrecera todas las respuestas relevantes. La academia y la teoria Importan si, como consideran los practicantes de los estudios culturales, una mejor comprension del mundo y la labor intelectual tienen su lugar en la transformacion del mundo. A hora bien esta reivindicación de la labor intelectual no significa que en los estudios culturales se fetichice la academia o la teoria en nombre de una supuesta superioridad epistemologica absoluta (propias del pensamiento eurocentrico). Una teorizacion de lo politico y una politizacion de 


\section{EdrabPestrepo}

Estudios culturales y educacion:

Posibilidades, urgencias y limitaciones, artículo de reflexión

lo teórico (G rossberg,2009), he ahí una formulación que atrapa ese otro rasgo infaltable en los estudios culturales: la voluntad politica.

Por todo lo argumentado, la más adecuada manera de concebir la especificidad de los estudios culturales es con la nocion de contextual ismo radical G Gossberg 2010). Varios son los planos donde operaria el contextual ismo radical. En el epistemologico, el contextualismo radical que definirila los estudios culturales consiste en lo que ya se refirio como un pensamiento sin garantias. Este pensamiento se funda en la nocion de articulacion, es decir, que las cosas del mundo (prácticas, entidades, ideas, etc.) son resultado de la relaciones que las constituyen. Pero estas relaciones son historicamente contingentes y situadas. El conjunto de articul aciones significativas para comprender la especificidad de una cosa en el mundo es la manera en que se define el contexto. El contexto no es el telón de fondo, el escenario donde al go sucede, sino sus condiciones de existencia y de transformacion.

En el plano teórico, el contextualismo radical de los estudios culturales refiere a que estos no son definidos por la utilizacion de una teoría de la cultura o del poder especifica, ni por la citacion de unos autores concretos (citar a Stuart Hall no garantiza que estemos en el terreno de los estudios culturales), sino que la teorizacion es un acto mundanal derivado las investigaciones concretas y empiricamente orientadas que establecen un constante forcejeo e interrupcion de losinsumos teoricos con los que se cuenta. Lafluidez en angelicales formul aciones teoricas con su concomitante violencia teórica (el mundo no existe sino como ilustración o corroboración de las teorías armadas de antemano), es lo opuesto del contextualismo radical en el plano teórico.

En el plano metodológico, es el pluralismo metodológico lo si se quiere un eclecticismo estrategico y sin disculpas) lo que guia el contextualismo radical en los estudios culturales. Esto se traduce en el principio de que las metodologías y las tecnicas de investigación son herramientas que hay que utilizar en funcion de las preguntas y competencias, sin descartar alguna por simple puritos 0 desautorizaciones disciplinares. Es cuestionar esoș limites metodologicos y de las tecnicas de investigacion que suelen constreñir a los practicantes de las disciplinas.' un antropólogo hace etnografía, siendo una cuasi herejía no hacerla', o un historiador se define en su autoridad y posibilidad de enunciación en su labor de archivo..., En tanto eclecticismo estratégico, los estudios culturales se darian licencias e introducirian irreverencias que difícil mente son posibles desde las posiciones y tradiciones disciplinarias.

En el plano político, los estudios culturales son un contextualismo radical en tanto no asumen que una práctica, una concepción o un sujeto tienen el mismo significado político por fuera del contexto que los constituye. Lo que puede operar como progresista en una situacion determinada, puede ser profundamente reaccionario en otra. Una practica emancipadora en un contexto, puede devenir 
Revista de

investigaciones UNAD

Volumen 10. Número 1. Junio 2011

en sujetadora en otro (0, más sugerente aún, puede ser sujetadora precisamente por su caracter emancipador), En suma, el contextualismo radical en el plano de o político no es una apelación al nihilismo, sino algo tan sencillo y compleio a la vez de que el significado político de al go no esta dado independientemente del contexto donde existe.

Finalmente, en el plano del proyecto de los estudios culturales también aplicalo del contextualismo radical en el sentido de que en su existencia estan profundamente situados, es decir, no pueden ni pretenden ser identicos en todas partes y momentos sino mas que adquirir una especificidad dependiendo de los contextos, no pueden dejar de ser un auténtico producto de los mismos.

En suma, hay que distinguir entre estudios culturales y estudios sobre la cultura porque los estudios culturales constituyen un proyecto intel ectual y politico que: 1) concibe la cultura-como-poder y el poder-como-cultural; 2) suponen un enfoque no reduccionisța que se expresa en una actitud transdisciplinaria; 3) implican una vocacion politica que busca intervenir sobre el mundo; y 4) su encuadre es, el contextualismo radical (con respecto a su forma de teorizacion, a las metodologias utilizadas, a su conceptualizacion de la politica y su propio proyecto).

El planteamiento de que existe un proyecto intelectual y político compartido que establecería una especificidad de los estudios culturales no significa que se considere que los estudios culturales son todos iguales. A contrario, los estudios culturales deben ser pensados como un terreno de divergencias y tensiones. Pero lo que podríamos ll amar la heterogeneidad constitutiva de, los estudios culturales, no significa que entonces no existan ciertos referentes basicos compartidos que hacen que no cualquier cosa quepa dentro de los estudios culturales.

En este punto se podrían establecer algunas confluencias entre el proyecto intelectual y politico descrito de los estudios cul turales y los estudios criticos de la educacion. Para empezar, pudiera argumentarse que los estudios críticos de la educacion también se diferenciarian de los estudios sobre la educacion a partir de una serie de criterios como una clara voluntad politica que se materializaria en el proposito de la comprension de la praxis educativa en un sentido amplio les decir, no restringido a la escuela) para contribuir en la transformación social. Gran parte del enorme trabaijo acumulado sobre educacion popular y pedagogia critica encajarian precisamente en ese proyecto intelectual y politico de los estudios criticos de la educacion.

Otro aspecto donde se vislumbran ciertas confluencias es la de pensar la practica pedagogica como un hecho a la vez cultural y político, por lo que nos encontrariamos en el terreno de la cultura-como-poder y el poder-como-cultural. Dos son los aspectos mas evidentes en los que esta confluencia puede ser pensada. Por un lado, la educacion requiere ser concebida como un proceso, experiencia, institucion que atane directamente a la cultura-como-poder y el poder-como- 


\section{EdracbPestrepo}

Estudios culturales y educación:

Posibilidades, urgencias y limitaciones, artículo de reflexión

cultural en tanto se reproducen-reelaboran-resisten modelos de sujeto y sociedad. Esto tambien es valido para abordar las concepciones mucho mas amplias de las practicas y procesos pedagogicos como han sido el aboradas desde la educacion popular por ejemplo. Por el otro, es relevante este enfoque si consideramos quelos educadores serian "trabajadores de la cultura" y en tal sentido "sujetos politicos" en el sentido amplio, situacion que no siempre se reconoce en la practica cotidiana.

Urgencias y limitaciones

Tal como han sido presentados, los estudios culturales suponen un proyecto crítico que es urgente en el contexto colombiano. Es urgente en el plano del establecimiento académico porque cada vez mas se consolida una tendencia hacia la elitización profesionalizante de las ciencias sociales en el pals en nombre de unas politicicas de ciencia y tecnología que apelan a las narrativas de la internacional izacion y de la calidad.

Internacionalización es una noción que, al menos en Colombia, circula con frecuencia en el ambito universitario y en las politicas de ciencia y tecnologia agenciadas por la entidad gubernamental (Colciencias), para referirse a una deseada e inevitable articulacion de la 'comunidad académica nacional' con la global. Deseada articul acion porque supone una suerte de 'elevacion' de la calidad de la ciencia y tecnologias del pals a unos 'niveles superiores' que se asumen son los que existen en el 'escenario internacional', Esta 'elevación' se considera consecuencia de una mayor exposicion y visibilidad de la ciencia y tecnología producida en el pais a los estandares de la 'comunidad internacional'. Por tanto, las narrativas de la internacionalizacion de la ciencia y tecnologia en el pals propugnan por una 'desprovincialización' en aras de una 'cosmopolitanizacion' de la labor cientifica y tecnologica.

También se asume la internacionalización como inevitable porque se la considera como un aspecto de la ineludible g lobalizacion a la que el pals se enfrenta. Desde esta perspectiva, ante el avance de la globalizacion no hay otra al ternativa que la de articularse 'eficientemente' a la 'comunidad global'. Entendida de esta manera, la internacionalizacion se asocia facilmente a las predicas celebratorias de la globalizacion. Estas predicas constituyen, desde una aparente neutralidad, narrativas teleologicas que presentan como necesario lo que es contingente e historico obliterando otros principios de inteligibilidad del presente. ${ }^{4}$

A hora bien, esto no es simplemente una inocua narrativa que embruja la imaginacion de la burocracia de la ciencia y la tecnología sino que en nombre de la internacionalizacion aśi concebida se adelanta una agresiva politica de normalización de las subjetividades y prácticas académicas en el país. La

4 De ahí que el antropólogo haitiano Michel-Rolph Trouillot (2003) denomine a este tipo de narrativas como globalototaritarismo y argumente la necesidad de desnaturalizarlas estudiándolas etnográficamente e historizando sus imaginarios. 
Revista de

investigaciones UNAD

Volumen 10. Número 1. Junio 2011

producción y circulación del conocimiento académico han sido objeto de toda una gubernametalizacion que esencialmente las reduce a una serie de indicadores y dispositivos de captura. Indicadores de ciencia y tecnologia, indexaciones de las revistas, formalización de grupos de investigación y fijación de la producción de os individuos en hojas de vida estandarizadas son a gunas de las intervenciones mas obvias que desde la entidad gubernamental (Colciencias) se adelantan cada vez con mayor impetu. El malestar existente entre al gunos academicos (sobre todo pertenecientes a las ciencias sociales y humanas) sobre los criterios y procedimientos de estas politicas de normalización no se ha consolidado en un movimiento de crítica visible y consistente', en gran parte porque dependen de instituciones (universidades, institutos, Ongs) que se han plegado sin mayor examen a estas políticas.

Estas políticas de ciencia y tecnología han ido ganando terreno articulándose con otras tendencias para ir academizando y profesionalizando (en el sentido de las estandarizaciones y normalizaciones indicadas) las universidades y otros centros de produccion de conocimiento. En el transcurso de veinte anos, se han ido transformando los terminos y las condiciones de la labor academica. Hoy predomina la logica de, la hoja de vida de los individuos y las instituciones en las que importan mas el número y el lugar de las publicaciones realizadas que lo que realmente esto puede significar social o politicamente, importan mas el numero y el lugar de obtencion de maestrias y doctorados que las cualidades de los doctores' para articularse con procesos relevantes politicamente, Hoy contamos cada vez más con una elite de doctores que investigan y publican febrilmente para posicionar sus carreras academicas.

L a urgenciadeunos estudios cul tural es quesupongan el proyecto crítico descrito en la primera parte de este texto, radica en su potencial irreverente y desestabil izador en el corazon mismo de la paulatina sedimentacion de un establecimiento académico elitista profundamente academizada y profesionalizada. Se requiere urgentemente de conștituir a los estudios culturales como un terreno estrategico dentro del establecimiento academico colombiano para problematizar la elitizacion de las ciencias sociales en el pais. A hora bien, esto no es tan sencillo ya que una de las mas grades limitaciones en el proyecto critico de los estudios culturales y sus posibles articulaciones con el campo de la educación radica en que en la actual institucionalizacion se impongan concepciones academizadas y banalizadas que reduzcan la voluntad politica de los estudios cultural es a un acercamiento textualista. El riesgo es que con su creciente institucional ización los estudios culturales pasen a significicar estudios sobre la cultura donde su vocacion politica se circunscriba, en el mejor de los escenarios, a un examen de relaciones de poder considerando que en eso consiste su intervencion.

Los estudios culturales tienen un lugar privilegiado para adelantar esta crítica porque son institucionalizados en universidades de elite como consecuencia de mismas las politicas de ciencia y tecnologia que habria que cuestionar. En el 


\section{EdracbPestrepo}

Estudios culturales y educación:

Posibilidades, urgencias y limitaciones, artículo de reflexión

imaginario de la burocracia académica y de amplios sectores de académicos, los estudios cul turales han sido celebrados porque supuestamente representan un campo interdisciplinar de punta y ampliamente exitoso en el establecimiento estadounidense que es su paradigma. Dado el fuerte colonialismo intelectual (Fals Borda 1987) con el que operan en Colombia las narrativas de la internacionalizacion, de la calidad y de la ciencia, el lugar de los estudios culturales es uno que podría incomodar más esos discursos y practicas que explican su propia institucionalizacion.

A quí se derivan una serie de confluencias entre el campo de los estudios culturales y el de la educación. Por un lado, habrian toda una serie de herramientás y estrategias derivadas del campo, de la educación que habria que considerar detenicamente para adelantar más adecuadamente el proceso de formacion de nuestros estudiantes de una forma que signifique la valoracion e incorporacion de este proyecto critico de los estudios culturales en su agenda y practica profesional. Esto es un reto descomunal porque gran parte de los estudiantes que llegan a los programas de maestria en estudios culturales existentes en el pais lo hacen por razones muy distintas a las que se encuentran identificados con un proyecto critico en general y han identificado en los estudios culturales una posibilidad particul ar, de articularlo, El grueso de nuestros estudiantes provienen de sectores económicamente privilegiados de la sociedad colombiana y muchos de ellos sin explicitas preocupaciones politicas, A socian estudios culturales con gestion cultural o con los estudios interdisciplinarios y contemporáneos sobre la cultura.

A partir de la institucionalización de los estudios culturales, entonces, se hace cada vez más indispensable un análisis, de las practicas de enseñanza, de los model os pedagogicos, el curricul o (explicito y, ocul to), los saberes pedagogicos que ocurren en los programas de formación en estudios culturales. Cabe preguntarse, por lo tanto, ¿que ocurre con el saber que se despliega en tanto practica de enseñanza en estudios culturales?

Por el otro lado, la relación delosestudios culturales con el campo de la educación se encuentra en la relevancia de las herramientas y conceptualizaciones adelantadas en este campo para potenciar la labor desestabilizadora de los estudios culturales en el establecimiento académico dominante. Siguiendo a Gramsci, la hegemonia consiste en una permanente disputa en el terreno de la sociedad civil (mediante lo que denomino guerra de posiciones) por constituir el liderazgo moral, cultural, económico y político de lo que deviene en un bloque historico. Gramsci referia al caracter educativo del proceso de configuracion y disputa de la hegemonia., Desde una perspectiva gramsciana, entonces, hegemonia no se reduce a coerción ni a imposicion por la fuerza como suele referirse comunmente con este termino, ni tampoco es algo moralmente perverso que debe ser denunciado y que pertenece naturalmente a las elites. Para Gramsci, al contrario, la lucha politica es una lucha por la hegemonia. 
Revista de

investigaciones UNAD

Volumen 10. Número 1. Junio 2011

Si los estudios culturales buscan desestabilizar el establecimiento académico elitizado colombiano requieren precisamente de pensar su labor en términos de disputa por la hegemonia, y ahí es precisamente donde el campo de la educacion y sobre todo de la pedagogia critica puede ofrecerle a los estudios culturales insumos conceptuales y metodologicos pertinentes. A hora bien, esta disputa por la hegemonia entendida en gran parte como labor pedagogica no solo debe ser orientada hacia los col egas en las diferentes areas de las ciencias sociales y humanidades, sino tambien con los col egas que se dicen practicantes de los estudios culturales. Esto ultimo es muy importante ya que la nocion de los estudios cultural es como proyecto intelectual y politico crítico no se expresa en gran parte de los docentes y practicantes de los estudios culturales. No son pocos los que se encuentran en estos programas por razones circunstanciales, desconociendo en gran parte las discusiones sobre la especificidad de los estudios culturales y sus implicaciones. La directora de uno de esos programas, por ejemplo, en una charla publica hacia recientemente una historia de los estudios culturales como un desplazamiento de los estudios disciplinares, pasando por los estudios tematicos a los estudios interdiscipl inares suponiendo que la novedad de los estudios cultural es radicaba precisamente en esta manera mas amplia' de abordar los problemas.

Hay otro aspecto de la urgencia de los estudios culturales en el contexto colombiano gue merece considerado, esta vez por fuera del establecimiento académico. Estoy pensando en como se entiende la labor intelectual en diferentes procesos organizativos como en el caso de los de los movimientos sociales articulados en torno a reivindicaciones etnicas, raciales, sexuales y de derechos humanos entre otros. En muchos de estos movimientos la labor intelectual se tiende a circunscribir a una constatación y celebración del discurso politico existente desde el que estan despl egando su practica politica. No son extranos en estos movimientos actitudes abiertamente anti-teoricistas y antiacademicistas.' se argumenta que la teoria y la academia son irrelevantes.para su propia practica, incl uso que son una traba porque desconoce el conocimiento de la gente el cual aparecería como más auténtico, profundo y verdadero. ${ }^{5}$

La urgencia de los estudios culturales consiste aquí en la articulación de una labor intelectual que sigue el precepto gramsciano del pesi mismo del intelecto, desde el optimismo de la voluntad. Esto significa que hay que comprender en lo concreto y de manera contextual como se estan amarrando las relaciones de poder y las practicas significativas, sin concesiones en nombre de lo que a uno le gustaría que fuesen las cosas. Pero esto va de la mano de una identificación y actividad politica desplegada más all a del simple ejercicio intelectual, U na labor intelectual orientada por una vol untad política permitiria un auténtico

5 Con el posicionamiento en el país de una academia cada vez más elitizada e irrelevante políticamente y la consecuente consolidación de una especie de nobleza académica, es entendible que desde los movimientos sociales se mire con recelo las esporádicas intromisiones de académicos que con un estilo pontificante y arrogante les indican sus errores y lo que deberían hacer. 


\section{EdracbPestrepo}

Estudios culturales y educación:

Posibilidades, urgencias y limitaciones, artículo de reflexión

diálogo con procesos organizativos de diversa índole. Cabe aclarar, sin embargo que este no es el único ambito en el que se despliega la vocacion pol itica de los estudios cultural es. Las disputas en el establecimiento academico antes indicadas es otro, pero tambien se puede identificar las politicas estatales, los programas de las, Ongs o las actividades empresariales como posibles terrenos de articul acion de urgencias de unos estudios culturales que pongan en evidencia la filigrana de las mas variadas practicas de gubernamental izacion que parecen pasar inadvertidas para el grueso de los academicos y expertos.

Nuevamente, las herramientas y conceptualizaciones derivadas de los estudios criticos de la educacion, apuntalarían y potenciarían la intervencion de los estudios culturales en el ámbito de los procesos organizativos. Y esto no solamente en el sentido de 'enseñar' lo que una labor intelectual, situada permite comprender, sino también en cuanto hay una dimensión pedagógica en el trabajo intelectual mismo tendiente a la comprension.

\section{Reflexiones finales}

Colonialidad del saber es un concepto que se ha elaborado desde una red de académicos latinoamericanos situados tanto en A merica Latina como en los Estados U nidos. El término sehaderivado del de colonialidad del poder sugerido por el sociólogo peruano Anibal Quijano hace unos veinte años. Una de las distinciones mas interesantes sugeridas, ha sido la distinción entre col onialismo y colonialidad. La colonialidad sería la logica que subyace al colonialismo, pero que se mantendría incluso hasta nuestros días, ya que se concibe como el lado oscuro e inmanente de la modernidad. ${ }^{6}$ La colonialidad del saber refiere a la dimension epistemica de la colonialidad del, poder, La colonialidad del saber es constituilda por un patron de clasificacion y jerarquizacion global de los conocimientos, donde unos aparecen como la encarnacion misma del conocimiento verdadero y que es mas adecuado, mientras que otros conocimientos son expropiados, inferiorizados y silenciados.

Retomando esta inspiradora conceptualización, me gustaría cerrar esta ponencia con la pregunta tanto para el proyecto intelectual y político de los estudios cul turales como para los estudios criticos de la educacion sobre esta dimension epistémica de la colonialidad del poder. Esto es, ihasta donde en estos dos campos se están reproduciendo o confrontando los efectos estructurantes de la colonialidad del saber? ¿estamos examinando con la suficiente densidad las practicas concretas que ambos campos agencian desde esta radical perspectiva de la diferencia colonial?

6 Para ampliar estos conceptos, así como las fuentes y algunas de las criticas adelantadas a esta corriente conocida como proyecto decolonial o grupo de la modernidad/colonialidad, ver Restrepo y Rojas (2010). 


\section{Revista de investigaciones UNAD \\ Volumen 10. Número 1. Junio 2011}

\section{Referencias bibliográficas}

Fals Borda, Orlando 1987. Ciencia propia y colonialismo intelectual : los nuevos rumbos. Bogotá: Carlos Valencia Editores.

Grossberg, Lawrence. 2010. Estudios culturales. Teoría, política y práctica. Valencia: Letra Capital.

Hall, Stuart. 2007. "Epilogue: through the prism of an intellectual life". Brian Meeks (ed.), Culture, Politics, Race and Diaspora. pp. 269-291 Kingston: Ian Randle Publishers.

. [1992] 2010. "Estudios culturales y sus legados teóricos". En: Sin garantías: Trayectorias y problemáticas en estudios culturales. pp. 51-71 Bogotá-Quitó-Lima: Envión Editores-Instituto Pensar-Universidad Andina-IEP.

. [1990] 2010. "Identidad cultural y diáspora”. En: Stuart Hall, Sin garantías. Trayectorias y problemáticas en estudios culturales. pp. 349-462. Popayán-Lima-Quito: Envión Editores-IEP-Instituto Pensar-Universidad Andina Simón Bolívar.

2009. El corazón de los estudios culturales. Tabula Rasa (10): 13-48.

Mato, Daniel 2002. "Estudios y otras prácticas latinoamericanas en cultura y poder". En: Daniel M ato (ed.), Estudios y otras prácticas latinoamericanas en cultura y poder. pp. 21-43 Caracas: CLACSO.

Restrepo, Eduardo y Axel Rojas. 2010. Inflexión decolonial: fuentes, conceptos y críticas. Popayán: Editorial Universidad del Cauca.

Richard, Nelly. 2001 "Globalización académica, estudios culturales y crítica latinoamericana" En Mato, Daniel (ed.), Estudios Latinoamericanos sobre cultura y transformaciones sociales en tiempos de globalización. pp. 185-199Buenos Aires: Clacso.

Trouillot, Michel-Rolph. 2003. Global Transformations. Anthropology and the Modern World. New York: Palgrave Macmillan.

Walsh, Catherine, Freya Schiwy y Santiago Castro-Gómez. 2002. Indisciplinar las ciencias sociales. Geopolíticas del conocimiento y colonialidad del poder. Perspectivas desde lo andino. Quito: Universidad Andina Simón Bolívar-Ediciones Abya-Yala. 